\title{
Study on essential oils from four species of Zhishi with gas chromatography-mass spectrometry
}

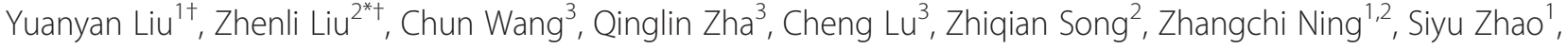 \\ Xinmiao $\mathrm{Lu}^{1}$ and Aiping $\mathrm{Lu}^{3,4^{*}}$
}

\begin{abstract}
Background: Citrus fruits are widely used as food and or for medicinal purposes, and they contain a host of active substances that contribute to health. The immature fruits of Citrus sinensis Osbeck and its cultivars (CS), C. junos Sieb. ex Tanaka (C), C. aurantium L. and its cultivars (CA) and Poncirus trifoliate Raf. (PT) are the most commonly used medicinal herbs in Traditional Chinese Medicine, called Zhishi. And their mature fruits can be used as food.

Results: In this study, the essential oils of four different Zhishi species were extracted by steam distillation and detected using gas chromatography- mass spectrometry (GC-MS). A total of 39 volatiles from the four species were tentatively identified. The limonene was the most abundant amongst the four species. Principal component analysis (PCA) of essential oils showed a clear separation of volatiles among CS, CJ and PT. However, CA could not be separated from these three species. Additionally, the volatiles accounting for the variations among the widely separated species were characterized through their corresponding loading weight.

Conclusion: Sesquiterpenes were identified as characteristic markers for PT. The content of some monoterpenes could be as taxonomic markers between CS and CJ. This work is of great importance for the evaluation and authentication of Zhishi samples through essential oils.
\end{abstract}

Keywords: Citrus fruits, Essential oils, GC-MS, PCA, Zhishi

\section{Background}

Citrus genus is the most important fruit tree crop in the world, with an annual production of approximately 102 million tons [1]. Citrus fruits are used in the food, cosmetic and pharmaceutical industry. They are consumed fresh and processed, as juices, jam, jellies, molasses, etc.. The fresh fruits are important starting materials for juice production [2]. Especially, chemical industry extracts from Citrus bioactive compounds like flavonoids, vitamins, dietary fiber and essential oils, etc. have beneficial effects on human health. The essential oils of Citrus fruits are widely used as flavoring in foods, perfumes and pharmaceutical formulations due to their functional properties like antimicrobial, antifungal, as well as analgesics,

\footnotetext{
* Correspondence: zhenli_liu@sina.com; lap64067611@126.com

${ }^{\dagger}$ Equal contributors

${ }^{2}$ Institution of Basic Theory, China Academy of Chinese Medical Sciences, Beijing 100700, China

${ }^{3}$ Institute of Basic Research in Clinical Medicine, China Academy of Chinese Medical Sciences, Beijing 100700, China

Full list of author information is available at the end of the article
}

cough suppressants, and expectorants for eliminating phlegm [3-8]. Citrus oils extraction methods include steam distillation [9], cold pressing [10] and static headspace solid-phase microextraction [11].

The immature fruits of $C A, C S, C J$ and $P T$ can be used as Zhishi, one of the primary traditional Chinese medicinal plants in China $[12,13]$. The herbs from different species and cultivation locations are widely used in clinical applications. Such herbs have been used to treat stuffiness, intestinal fullness sensation and distending pain, diarrhea and dysentery in addition to the retention and preservation of food [14]. However, the efficacy of these species of Zhishi for treating digestive disturbances varies due to differences in the types and quantities of the chemical substances they contain $[15,16]$. Our previous studies based on four flavanone compounds demonstrate that compounds from different species of Zhishi, such as $C A, C S$ and $C J$, can be distinguished by established differential modes of acquisition [17]. In addition to flavanones, essential oils are an important type of pharmacodynamic substance in Zhishi. Therefore, it is
() ChemistryCentral 
important to elucidate the differences between volatile substances among different species. Numerous studies [18-23] on the essential oils of Zhishi have been conducted using GC and GC-MS. However, because there is still a lack of information regarding the essential oils of different species of Zhishi, a comparative study is necessary.

Therefore, in this study, 75 authentic Zhishi samples from four species of Citrus fruit (CA, CS, CJ and PT) were collected. The essential oils were obtained by using steam distillation, and then were separated and identified by GC-MS. In total, 39 volatiles were tentatively identified and quantified, and among them were 26 monoterpenes, 8 sesquiterpenes, and 5 esters, aliphatic alcohols and aliphatic aldehydes. The details were shown in the Additional file 1. Then, a multivariate statistical analysis method was employed to elucidate the variation of volatile substances among different species of Zhishi. This is the first report on comparison study of essential oils from these four Zhishi species.

\section{Results and discussion \\ Quantification of the volatile components}

In total, 39 compounds were tentatively identified, constituting approximately $85.14-100 \%$ of the entire volatile concentration (Table 1). Each compound was identified by matching its retention characteristics and MS fragmentation patterns against standards and library spectra. The results are expressed as relative weight percentages calculated from peak areas. Volatiles were categorized into five chemical groups consisting of monoterpenes, sesquiterpenes, esters, aliphatic alcohols and aliphatic aldehydes. For all of the four Citrus species, the total content of essential oils primarily consisted of 26 monoterpenes, which accounted for $52.17-100 \%$ of the entire volatile concentration. The other four chemical groups sesquiterpenes, esters, aliphatic alcohols and aliphatic aldehydes - contained in $C A, C S$ and $C J$ had minor contributions to the entire volatile concentration accounting for only $0-2.28 \%$. Sesquiterpenes were present in the oils analyzed in the greatest amount (3.8-34.28\%) in the $P T$ species compared to the other three species. This result is not surprising as sesquiterpenes hydrocarbons are important in the characteristic aroma of many kinds of Citrus fruits [24]. Alpha and $\beta$-pinene are chemically unstable bicyclic terpenes due to a strained four membered ring. Thus, they are found at low levels and evenly distributed in all the four species.

\section{GC-MS analyses of the four different species of Zhishi samples}

In the 35 samples of $C A$, monoterpenes produced the highest percentage of volatiles (84.67-99.61\%). Among the volatiles, the most predominant was limonene (27-82.84\%,
Mean $63.47 \%)$, followed by $\gamma$-terpinene $(0.25-32.31 \%$, Mean 12.01\%), $\beta$-linalool (0-26.15\%, Mean 9.10\%), $\beta$ pinene $(0.4-17.01 \%$, Mean 2.59\%), $\beta$-cis-ocimene $(0-$ 9.99\%, Mean 2.21\%) and (+)-sabinene (0.1-8.26\%, Mean $1.77 \%)$. Meanwhile, the mean values of volatiles such as $\alpha-$ pinene, $\beta$-myrcene, $\mathrm{p}$-mentha-1,4(8)-diene, o-cymene, $(-)$-terpinene-4-ol and $\alpha$-terpieol were higher than $0.5 \%$, with an average percentage of total volatile concentration of $1.25 \%, 1.05 \%, 0.66 \%, 0.63 \%, 0.61 \%$ and $0.60 \%$, respectively. Other chemicals, such as germacrene $D, \alpha$-methyl$\alpha$-[4-methyl-3-pentenyl]-oxiranemethanol and n-octanal, belonging to sesquiterpenes, aliphatic alcohols and aliphatic aldehydes chemical groups, were minor volatile components in $C A$ species, averaging $0.26 \%, 0.35 \%$ and $0.33 \%$ of the total percentage, respectively.

For $C S$ species, monoterpenes were also the highest concentration of the volatile, averaging $85.3-100 \%$ of the total percentage. Limonene was the most abundant monoterpene in this species accounting for 72.75$89.35 \%$, with an average value at $82.25 \%$. Additionally, $(+)$-sabinene and $\beta$-linalool were recorded at a relatively lower proportion, $3.61-12.82 \%$ and $1.34-9.21 \%$, respectively. Other compounds within the monoterpenes group, such as $\beta$-myrcene, $\gamma$-terpinene, (-)-terpinene-4-ol, $\beta$ and $\alpha$-pinene, also occurred at a higher proportion, the mean amounts were $1.14 \%, 0.91 \%, 0.73 \%, 0.62 \%$ and $0.50 \%$, respectively. Compounds of other chemical groups had a minor amount, and the mean values were lower than $0.1 \%$.

For $C J$ species, the total amounts of monoterpene compounds ranged from $89.31-99.81 \%$. The most two abundant essentials, limonene and $\gamma$-terpinene accounted for 35.53-60.60\% (Mean 46.47\%) and 21.71-46.74\% (Mean 34.16), respectively. Other monoterpenes, such as $\beta$ linalool, $\beta$-pinene, $\alpha$-pinene, $\mathrm{p}$-mentha-1,4(8)-diene, ocymene, $\alpha$-thujene, $\beta$-myrcene, $(+)$-4-carene, $\alpha$-terpieol, p-cymen-2-ol and (+)-sabinene, each had average amounts higher than $0.5 \%$ at $3.53 \%, 2.80 \%, 2.61 \%, 1.63 \%, 1.51 \%$, $1.12 \%, 0.94 \%, 0.88 \%, 0.60 \%, 0.55 \%$ and $0.54 \%$, respectively. Except for germacrene D, which had an average value at $0.15 \%$ and belongs to the sesquiterpenes, compounds of other chemical groups were less than $0.1 \%$.

Interestingly, in the $P T$ species, sesquiterpenes (3.80$34.28 \%$ ) were an important chemical group of character impact volatiles because of the relatively high percentage in the overall volatile composition compared to the other three species. In addition, it serves a characteristic aroma for many types of Citrus fruits. Among these sesquiterpenes, the most predominant was caryophyllen (Mean $5.32 \%)$, followed by $\gamma$-elemene (Mean 3.81\%), $\beta$-farnesene (Mean 2.80\%) and germacrene D (Mean 1.83\%). Limonene and $\beta$-myrcene were the major monoterpenes in this species as the average value accounted for up to $48.14 \%$ and $18.29 \%$, respectively. 
Table 1 Identifications of Zhishi volatiles and their relative total ion current peak area from four different species

\begin{tabular}{|c|c|c|c|c|c|c|c|c|c|c|}
\hline \multirow[b]{2}{*}{ No. } & \multirow[b]{2}{*}{ Name } & \multirow[b]{2}{*}{ RI } & \multicolumn{2}{|l|}{$C A-1 \sim C A-35$} & \multicolumn{2}{|l|}{ CJ-1 CJ-15 } & \multicolumn{2}{|l|}{$C S-1 \sim C S-21$} & \multicolumn{2}{|l|}{$P T-1 \sim P T-4$} \\
\hline & & & $\begin{array}{l}\text { Relative } \\
\text { concentration }\end{array}$ & Mean & $\begin{array}{l}\text { Relative } \\
\text { concentration }\end{array}$ & Mean & $\begin{array}{l}\text { Relative } \\
\text { concentration }\end{array}$ & Mean & $\begin{array}{l}\text { Relative } \\
\text { concentration }\end{array}$ & Mean \\
\hline 1 & a-Thujene & 943 & $0-1.15 \%$ & $0.40 \%$ & $0.35-1.71 \%$ & $1.12 \%$ & $0-0.09 \%$ & $0.03 \%$ & 1 & 1 \\
\hline 2 & a-Pinene & 948 & $0.28-2.97 \%$ & $1.25 \%$ & $0.88-3.97 \%$ & $2.61 \%$ & $0.25-0.75 \%$ & $0.50 \%$ & $0-1.52 \%$ & $0.58 \%$ \\
\hline 3 & (+)-Sabinene & 979 & $0.1-8.26 \%$ & $1.77 \%$ & $0-2.21 \%$ & $0.54 \%$ & $3.61-12.82 \%$ & $7.72 \%$ & $0.55-2.54 \%$ & $1.35 \%$ \\
\hline 4 & $\beta$-Pinene & 982 & $0.4-17.01 \%$ & $2.59 \%$ & $1.64-3.96 \%$ & $2.80 \%$ & $0.35-1.01 \%$ & $0.62 \%$ & $0-3.46 \%$ & $1.83 \%$ \\
\hline 5 & $\beta$-Myrcene & 992 & $0-2.72 \%$ & $1.05 \%$ & $0.55-1.55$ & $0.94 \%$ & $0-1.51 \%$ & $1.14 \%$ & $8.60-28.91 \%$ & $18.29 \%$ \\
\hline 6 & a-Phellandrene & 1004 & $0-0.42 \%$ & $0.05 \%$ & $0.04-0.37 \%$ & $0.09 \%$ & $0-0.05 \%$ & $0.02 \%$ & $0.84-9.00 \%$ & $4.74 \%$ \\
\hline 7 & (+)-4-Carene & 1015 & $0-0.88 \%$ & $0.33 \%$ & $0.54-1.23 \%$ & $0.88 \%$ & $0.08-0.35 \%$ & $0.17 \%$ & $0-0.18$ & $0.09 \%$ \\
\hline 8 & o-Cymene & 1022 & $0-5.86 \%$ & $0.63 \%$ & $0.38-4.96 \%$ & $1.51 \%$ & $0-0.22 \%$ & $0.03 \%$ & $0-0.23 \%$ & $0.11 \%$ \\
\hline 9 & Limonene & 1026 & $27-82.84 \%$ & $63.47 \%$ & $35.53-60.60 \%$ & $46.47 \%$ & $72.75-89.35 \%$ & $82.25 \%$ & 21.19-69.19\% & $48.14 \%$ \\
\hline 10 & $\beta$-trans-Ocimene & 1033 & $0-0.66 \%$ & $0.06 \%$ & $0-0.57 \%$ & $0.06 \%$ & $0-1.09 \%$ & $0.15 \%$ & $0-0.57 \%$ & $0.15 \%$ \\
\hline 11 & $\beta$-cis-Ocimene & 1042 & $0-9.99 \%$ & $2.21 \%$ & $0-0.89 \%$ & $0.38 \%$ & $0-1.02 \%$ & $0.31 \%$ & $1.46-4.96 \%$ & $2.60 \%$ \\
\hline 12 & y-Terpinene & 1052 & $0.25-32.31 \%$ & $12.01 \%$ & $21.71-46.74 \%$ & $34.16 \%$ & $0.31-2.63 \%$ & $0.91 \%$ & $0-0.73 \%$ & $0.40 \%$ \\
\hline 13 & cis- $\beta$-Terpineol & 1060 & $0-0.52 \%$ & $0.06 \%$ & $0-0.13 \%$ & $0.05 \%$ & $0-0.29 \%$ & $0.12 \%$ & 1 & $\backslash$ \\
\hline 14 & p-Mentha-1,4(8)-diene & 1081 & $0.07-1.79 \%$ & $0.66 \%$ & $0.95-2.33 \%$ & $1.63 \%$ & $0-0.15 \%$ & $0.08 \%$ & $0-0.12 \%$ & $0.05 \%$ \\
\hline 15 & $\beta$-Linalool & 1089 & $0-26.15 \%$ & $9.10 \%$ & $0.73-7,95 \%$ & $3.53 \%$ & $1.34-9.21 \%$ & $4.13 \%$ & $0.37-1.18 \%$ & $0.66 \%$ \\
\hline 16 & $\begin{array}{l}\text { trans-1-methyl-4-(1-methylethyl)-2- } \\
\text { Cyclohexen-1-ol }\end{array}$ & 1112 & $0-1.1 \%$ & $0.04 \%$ & 1 & 1 & $0-0.05 \%$ & 1 & $0-0.21 \%$ & $0.07 \%$ \\
\hline 17 & $\begin{array}{l}\text { cis-1-methyl-4-(1-methylethyl)-2- } \\
\text { Cyclohexen-1-ol }\end{array}$ & 1129 & $0-0.07 \%$ & 1 & 1 & 1 & 1 & 1 & $0-0.12 \%$ & $0.03 \%$ \\
\hline 18 & $\beta$-Citronellal & 1141 & $0-0.2 \%$ & $0.04 \%$ & $0-0.05 \%$ & $0.01 \%$ & $0-0.06 \%$ & $0.02 \%$ & 1 & $\backslash$ \\
\hline 19 & (-)-Terpinen-4-ol & 1164 & $0-3.23 \%$ & $0.61 \%$ & $0.21-1.02 \%$ & $0.48 \%$ & $0.33-2.17 \%$ & $0.73 \%$ & $0-2.42 \%$ & $0.88 \%$ \\
\hline 20 & a-Terpieol & 1176 & $0-1.93 \%$ & $0.60 \%$ & $0.30-0.85 \%$ & $0.60 \%$ & $0.08-0.63 \%$ & $0.30 \%$ & $0-2.10 \%$ & $0.75 \%$ \\
\hline 21 & cis-Carveol & 1202 & $0-.095 \%$ & $0.03 \%$ & 1 & 1 & 1 & 1 & 1 & $\backslash$ \\
\hline 22 & $\beta$-Citronellol & 1210 & $0-0.48 \%$ & $0.06 \%$ & $0-0.04 \%$ & 1 & $0-0.03 \%$ & 1 & $0-0.30 \%$ & $0.08 \%$ \\
\hline 23 & $\beta$-Citral & 1224 & $0-0.72 \%$ & $0.06 \%$ & $0-0.04 \%$ & 1 & $0-0.21 \%$ & $0.11 \%$ & 1 & $\backslash$ \\
\hline 24 & trans-Geraniol & 1236 & $0-1.16 \%$ & $0.05 \%$ & 1 & 1 & $0-0.20 \%$ & $0.02 \%$ & 1 & $\backslash$ \\
\hline 25 & a-Citral & 1251 & $0-0.35 \%$ & $0.04 \%$ & $0-0.03 \%$ & $0.01 \%$ & $0-0.24 \%$ & $0.12 \%$ & 1 & $\backslash$ \\
\hline \multirow[t]{2}{*}{26} & p-Cymen-2-ol & 1269 & $0-1.29 \%$ & $0.11 \%$ & $0-1.23 \%$ & $0.55 \%$ & 1 & 1 & 1 & 1 \\
\hline & Monoterpenes (Total) & & $84.67-99.61 \%$ & $96.88 \%$ & $89.31-99.81 \%$ & $98.42 \%$ & $85.30-100 \%$ & $99.48 \%$ & $52.17-96.06 \%$ & $80.80 \%$ \\
\hline 27 & n-Octanal & 1002 & $0-0.87 \%$ & $0.33 \%$ & $0-0.27 \%$ & $0.08 \%$ & $0-0.22 \%$ & $0.09 \%$ & 1 & $\backslash$ \\
\hline \multirow[t]{2}{*}{28} & n-Decanal & 1191 & $0-0.52 \%$ & $0.06 \%$ & $0-0.07 \%$ & $0.01 \%$ & $0-0.13 \%$ & $0.02 \%$ & 1 & $\backslash$ \\
\hline & Aliphatic Aldehydes (Total) & & $0-1.39 \%$ & $0.39 \%$ & $0-0.38 \%$ & $0.09 \%$ & $0-0.35 \%$ & $0.11 \%$ & 1 & $\backslash$ \\
\hline 29 & 1-Octanol & 1064 & $0-0.21 \%$ & $0.02 \%$ & 1 & 1 & $0-0.04 \%$ & 1 & $0-0.03 \%$ & $0.01 \%$ \\
\hline \multirow[t]{2}{*}{30} & $\begin{array}{l}\text { a-Methyl-a-[4-methyl-3-pentenyl] } \\
\text { oxiranemethanol }\end{array}$ & 1065 & $0.04-1.26 \%$ & $0.35 \%$ & $0-0.38 \%$ & $0.11 \%$ & 1 & 1 & 1 & $\backslash$ \\
\hline & Aliphatic Alcohols (Total) & & $0.04-1.26 \%$ & $0.37 \%$ & $0-0.38 \%$ & $0.11 \%$ & $0-0.04 \%$ & 1 & $0-0.03 \%$ & $0.01 \%$ \\
\hline \multirow[t]{2}{*}{31} & Nerol acetate & 1334 & $0-0.17 \%$ & $0.03 \%$ & 1 & 1 & 1 & 1 & $0-0.55 \%$ & $0.14 \%$ \\
\hline & Esters (Total) & & $0-0.17 \%$ & $0.03 \%$ & 1 & 1 & 1 & 1 & $0-0.55$ & $0.14 \%$ \\
\hline 32 & $\beta$-Elemene & 1362 & $0-0.21 \%$ & $0.01 \%$ & 1 & 1 & 1 & 1 & $0-0.96 \%$ & $0.27 \%$ \\
\hline 33 & Caryophyllene & 1387 & 1 & 1 & 1 & 1 & 1 & 1 & $0-14.7 \%$ & $5.32 \%$ \\
\hline 34 & $\beta$-Farnesene & 1418 & 1 & 1 & $0-0.15 \%$ & $0.03 \%$ & $0-0.14 \%$ & $0.01 \%$ & $0.22-8.94 \%$ & $2.80 \%$ \\
\hline 35 & Germacrene D & 1442 & $0-0.99 \%$ & $0.26 \%$ & $0-0.55 \%$ & $0.15 \%$ & 1 & 1 & $0.49-4.75 \%$ & $1.83 \%$ \\
\hline 36 & $\begin{array}{l}\text { 4-isopropylidene-1-vinyl-o-Menth- } \\
\text { 8-ene }\end{array}$ & 1452 & $0-0.08 \%$ & $0.01 \%$ & $0-0.05 \%$ & $0.01 \%$ & 1 & 1 & $0-0.25 \%$ & $0.08 \%$ \\
\hline
\end{tabular}


Table 1 Identifications of Zhishi volatiles and their relative total ion current peak area from four different species (Continued)

\begin{tabular}{|c|c|c|c|c|c|c|c|c|c|c|}
\hline 37 & (Z,E)-a-Farnesene & 1462 & $0-0.05 \%$ & 1 & $0-0.08 \%$ & $0.01 \%$ & $0-0.03 \%$ & 1 & $0-1.31 \%$ & $0.33 \%$ \\
\hline 38 & $\gamma$-Elemene & 1493 & $0-0.19 \%$ & $0.01 \%$ & 1 & 1 & 1 & 1 & $0.37-10.60 \%$ & $3.81 \%$ \\
\hline \multirow[t]{2}{*}{39} & a-Sinensal & 1567 & 1 & 1 & 1 & 1 & $0.01-0.25 \%$ & $0.01 \%$ & 1 & 1 \\
\hline & Sesquiterpenes (Total) & & $0-1.32 \%$ & $0.29 \%$ & $0-0.75 \%$ & $0.20 \%$ & $0-0.71 \%$ & $0.01 \%$ & $3.8-34.28 \%$ & $14.44 \%$ \\
\hline
\end{tabular}

Listed values are the percentage of the total peak area for each species. ' '' Not detectable. The detailed data of the 75 samples from the four species is listed in the Additional file 1.

\section{Principal component analysis}

The variability of essential oils in the four Citrus species of Zhishi shown in Table 1 was not anticipated. Principal component analysis (PCA) was employed to examine this large data set without having to assign classifications before analysis. PCA is an unsupervised clustering method that requires little prior knowledge of the data set and acts to reduce the dimensionality of multivariate data without losing important information. Par-scaled (scaled to square root of standard deviation) mathematical methods were performed to pre-treat the data set resulting from the volatiles of different Citrus species. The multivariate statistical analysis methodology helps to identify inherent patterns in the data in an unbiased manner and highlights the similarities and differences amongst samples. This methodology also helps identify those essential oils that are the most representative within the entire data set.

\section{PCA score plot}

As shown in Figure 1, each coordinate represents a sample, and the separation of the four different species of Zhishi samples was observed in the PCA scores plot. A

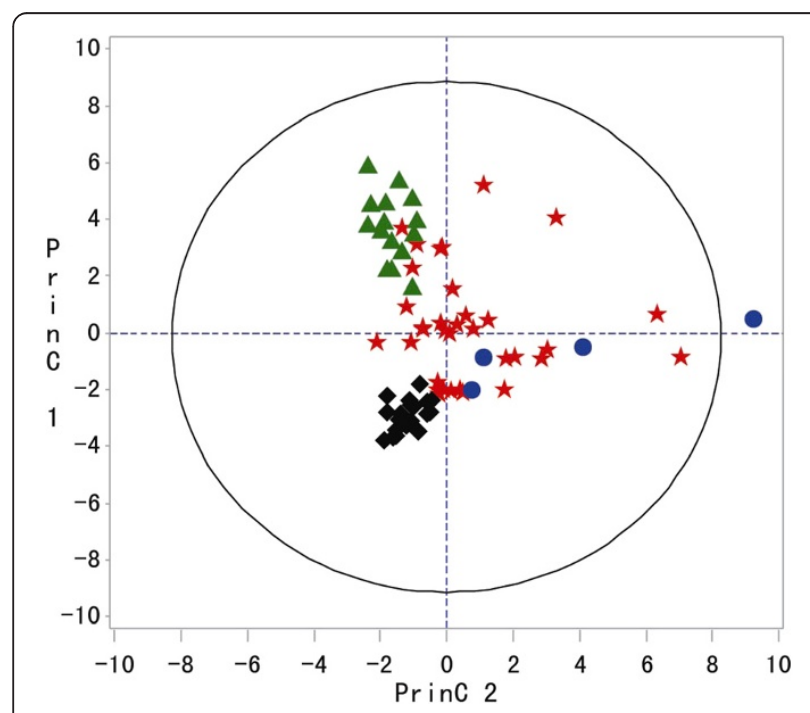

Figure 1 PCA scores plot (PC1 vs. PC2) of the four species of Zhishi samples: 35 samples from $C A, 15$ samples from $C J, 21$ samples from $C S$ and 4 samples from $P T$. (CA = (red star) $C J=$ (green triangle), $C S=$ (black diamond) and (PT = blue circle)). two-component PCA model cumulatively accounted for $54.5 \%$ of the total variance. The $C A$ species could not be separated from the other three species; however, according to our previous studies [17], $C S, C J$ and $C A$ could be differentiated from one other by choosing flavanones as marker compounds. The high degree of clustering and minimal overlap of CS, CJ and PT suggest that these three species have volatile profiles that are unique to each cultivar.

These three species, CS, CJ, and PT, were further analyzed by PCA. We observed three widely separated clusters in Figure 2 consisting of CS, CJ and PT. The PT samples were clearly separated from $C S$ and $C J$ by principal component 2 (PC2), whereas the $C J$ and $C S$ samples were clearly separated by principal component 1 (PC1). The results confirmed that $C S, C J$ and $P T$ were different regarding the levels and occurrence of these essential oils. However, the volatiles in $C A$ could not be distinguished from the other three species.

\section{PCA loading plot}

The corresponding PCA loading plot was utilized to identify the differential volatiles accountable for the separation among CS, CJ and PT. Although some information of low

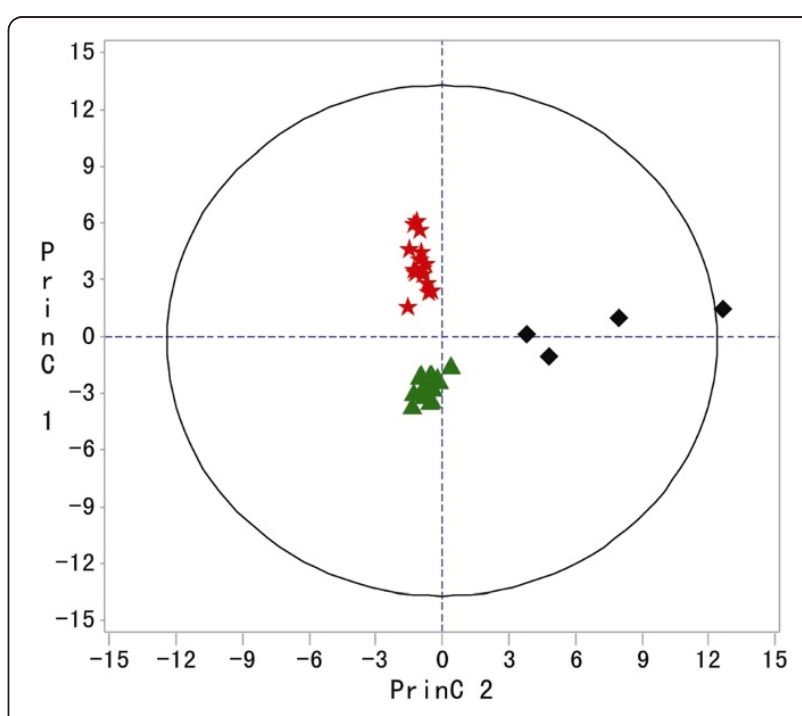

Figure 2 PCA scores plot (PC1 vs. PC2) of the three species of Zhishi samples: 15 samples from $C J, 21$ samples from CS and 4 samples from $P T$. $(C J=$ (red star), $C S=$ (green triangle) and $P T=($ black diamond $))$. 
intensity might be lost due to Par scaling, the loading plot clearly facilitated the profiling of the volatiles in our experiments. The volatiles that accounted for maximum variance in the data set are given more weight or loading. As shown in Figure 3, the variation within PC2 in the loading plot was due to the marker volatiles that accounted for the differences between $P T$ and the others as observed in the scores plot. Analogously, the distribution in PC1 of the loadings plot demonstrated the variation of $C S$ and $C J$, respectively. For example, sesquiterpenes, such as germacrene D (0.34 on PC2), caryophyllen (0.33 on PC2), $\beta$-myrcene (0.32 on PC2), $\beta$-elemene $(0.31$ on $\mathrm{PC} 2)$ and $\alpha$-farnesene $(0.27$ on $\mathrm{PC} 2)$ were the most differentiating volatiles, which played an important role in differentiating $P T$ from the CS and CJ. Meanwhile, in PC1, monoterpene compounds, such as p-mentha-1, 4(8)-diene (loading of $0.29)$, limonene (0.28), (+)-4-carene (0.28), $\beta$-pinene (0.28), $\gamma$-terpinene (0.28), $\alpha$-thujene (0.27), p-cymen-2-ol $(0.26)$ and $\alpha$-pinene (0.26), appeared to be of some relevance to the observed variability between $C S$ and $C J$.

\section{Discussion}

Literature reveals that limonene and $\gamma$-terpinene were the major components of the Citrus oils obtained by steam distillation and cold pressing [23]. The essential oils of four different species of Zhishi samples have been well studied and the major chemical groups are similar to the previous studies [21-23]. Limonene as a major constituent in several citrus oils (orange, lemon, mandarin, lime and grapefruit) is identified as the most predominant compound in the four species of Zhishi samples. The chemical compositions and their content

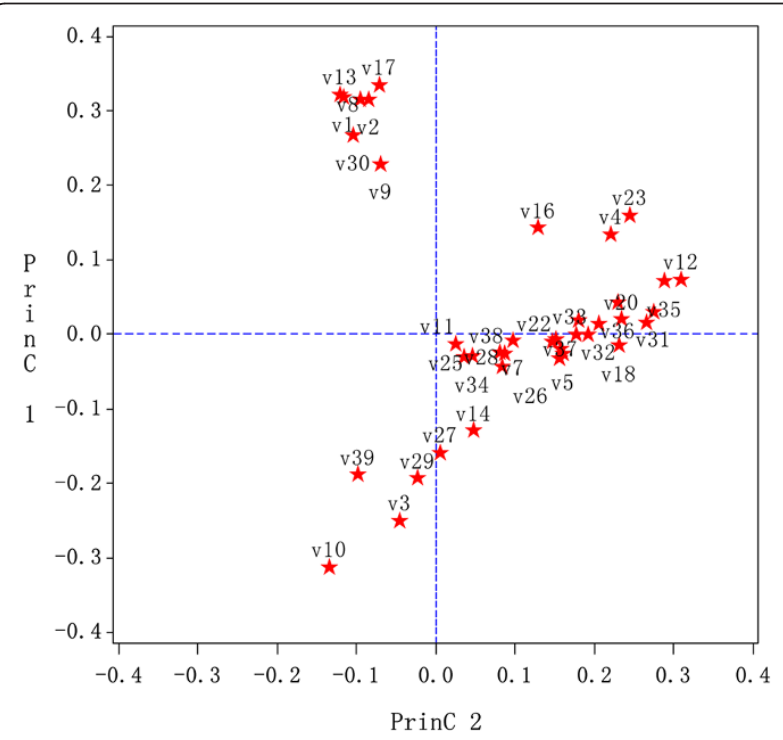

Figure 3 Overall PCA loading plot of the GC-MS signals associated with the three species of Zhishi samples: 15 samples from $C J, 21$ samples from $C J$ and 4 samples from PT. of the essential oils are varied in different species. Especially, sesquiterpenes were present in the oils analyzed in the greatest amount in the $P T$ species compared to the other three species. The contents of some monoterpene compounds such as p-mentha-1,4(8)-diene, limonene, $(+)$-4-carene, $\beta$-pinene, $\gamma$-terpinene, $\alpha$-thujene, $p$-cymen2 -ol and $\alpha$-pinene could be used as taxonomic markers between $C S$ and $C J$. The volatiles in $C A$ could not be distinguished from the other three species. This may be because that $C A$ samples distributed in a wide regions in China (Jiangxi, Hunan, Sichuan and Zhejiang). In the future, the active constituents contained in Zhishi samples from different regions need to be further investigated. Considering that all of the samples were collected in May or June under the same conditions impact of the growth stage factors on essential oil composition variability has been excluded.

\section{Experimental}

Plant material

All of the 75 Zhishi samples were collected from the main Zhishi-producing provinces of China: Jiangxi, Sichuan, Zhejiang, Fujian and Guizhou in May or June during a three-year period. For each of the 75 Zhishi samples, the species, local name, collection location, year of collection and growing environment are listed in Table 2. The diameters of collected Zhishi samples were limited to $0.5 \mathrm{~cm} \sim 2.5 \mathrm{~cm}$, which is consistent with the requirements of the Chinese Pharmacopoeia (Chinese Pharmacopoeia, 2010). Professors Ge Fei, Yan Zhuyun, Zhang Yungui, Xu Jianguo and Ke Fuzhi identified them as genuine samples of $C$. aurantium L. and its cultivars $(C A), C$. sinensis Osbeck and its cultivars $(C S), C$. junos Sieb. ex Tanaka $(C J)$ and Poncirus trifoliate Raf. (PT). The dried specimens (marked as $C A-1 \sim C A-35, C S-1 \sim$ CS-21, CJ-1 CJ-15 and PT-1 PT-4) were deposited at the Institute of Basic Theory, China Academy of Chinese Medical Sciences, Beijing, P. R. China. These four PT samples were wild growing.

\section{Extraction of essential oils}

Fresh, immature fruits of Zhishi were cut and dried in a manner similar to that described in the Pharmacopoeia of China [14]. The dried fruits were soaked in water for 2 hours and steam-distilled in a clevenger-type apparatus for 8 hours. This procedure was repeated 6 times. The oils were then dried over anhydrous sodium sulfate, diluted by acetone, and stored at $4-6^{\circ} \mathrm{C}$ before analysis.

\section{GC-MS analysis}

GC-MS analysis was performed on GCMS-QP2010 Plus (Shimadzu, Kyoto) equipped with a capillary column (Rxi-5 ms, $30 \mathrm{~m} \times 0.25 \mathrm{~mm}, 0.25 \mu \mathrm{m}$ ). Helium was used as the carrier gas at a flow rate of $1.0 \mathrm{~mL} / \mathrm{min}$. Oven 
Table $\mathbf{2}$ The origins of the $\mathbf{7 5}$ Zhishi samples collected

\begin{tabular}{|c|c|c|c|c|}
\hline No. & Species & Local name & Location and collection time & Growing environment \\
\hline \multicolumn{5}{|c|}{ Citrus aurantium L. } \\
\hline$C A-1 \sim 3$ & CA cv Xiucheng & Xiucheng & Xingan, Jiangxi; 2011 & 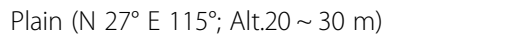 \\
\hline$C A-4 \sim 5$ & $C A \times P$. trifoliata & Citrange & Yuanjiang, Hunan; 2010 & 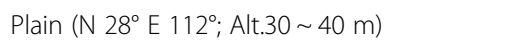 \\
\hline CA-6 $\sim 9$ & $C A$ & Sour orange & Jiangjin, Sichuan; 2010-2012 & Hillsides (N 29 E 106; Alt.229 m) \\
\hline$C A-10$ & CA cv Daidai & Daidai & Jiangjin, Sichuan; 2012 & Field margins (N 29 E 106 ; Alt.200 $230 \mathrm{~m}$ ) \\
\hline$C A-11 \sim 19$ & CA cv Xiucheng & Xiucheng & Xingan, Jiangxi; 2012 & 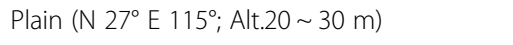 \\
\hline$C A-20 \sim 24$ & CA cv Xiucheng & Xiucheng & Zhangshu, Jiangxi; 2012 & Plain (N 27 E $115^{\circ}$; Alt.20 30 m) \\
\hline CA-25 & CA cV Jizicheng & Jizicheng & Zhangshu, Jiangxi; 2012 & 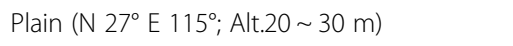 \\
\hline$C A-26$ & CA cv Daidai & Daidai & Huangyan, Zhejiang; 2012 & 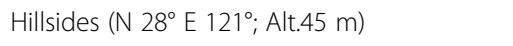 \\
\hline$C A-27$ & CA cv Morocco sour orange & Morocco sour orange & Huangyan, Zhejiang; 2012 & 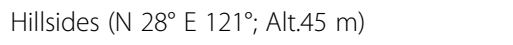 \\
\hline$C A-28 \sim 35$ & CA cv Xiucheng & Xiucheng & Xingan, Jiangxi; 2013 & 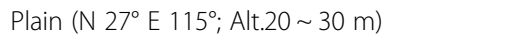 \\
\hline \multicolumn{5}{|c|}{ Citurs junos Sieb.ex Tanaka } \\
\hline $\mathrm{CJ}-1 \sim 3$ & CJ & Xiangcheng & Xingan, Jiangxi; 2011 & 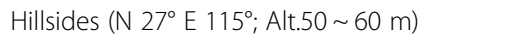 \\
\hline CJ-4 & CJ & TuanyeXiangcheng & Jiangjin, Sichuan; 2012 & 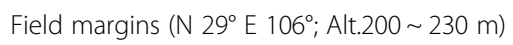 \\
\hline CJ-5 $\sim 10$ & CJ & Xiangcheng & Xingan, Jiangxi; 2012 & 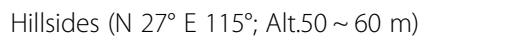 \\
\hline CJ-11 $\sim 15$ & CJ & Xiangcheng & Xingan, Jiangxi; 2013 & 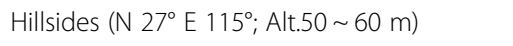 \\
\hline \multicolumn{5}{|c|}{ Citrus sinensis Osbeck } \\
\hline CS-1 & CS cv Jin Cheng & Jin Cheng & Qinglong, Guizhou; 2011 & 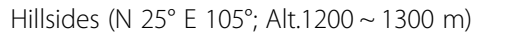 \\
\hline CS-2 & CS & Navel Orange & Xingan, Jiangxi; 2011 & 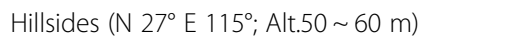 \\
\hline CS-3 & CS cv Jin Cheng & Jin Cheng & Qinglong, Guizhou; 2011 & 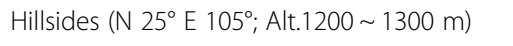 \\
\hline CS-4 & CS cv Navel orange & Navel orange & Qinglong, Guizhou; 2011 & 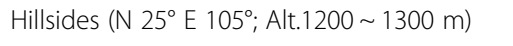 \\
\hline CS-5 & CS cv Blood orange & Blood orange & Qinglong, Guizhou; 2011 & 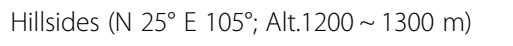 \\
\hline CS-6 & CS cv Valencia & Valencia & Qinglong, Guizhou; 2011 & 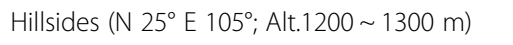 \\
\hline CS-7 & CS cv Blood orange & Blood orange & Jiangjin, Sichuan; 2012 & Hillsides (N 29 E 106; Alt.238 m) \\
\hline CS-8 & CS cv Valencia & Valencia & Jiangjin, Sichuan; 2012 & Hillsides (N 29 E 106; Alt.238 m) \\
\hline CS-9 & CS cv Navel orange & Navel orange & Jiangjin, Sichuan; 2012 & Hillsides (N 29 E 106; Alt.238 m) \\
\hline CS-10 & CS cv Peng an 100 & Peng an 100 & Jiangjin, Sichuan; 2012 & 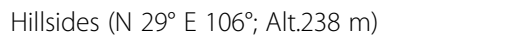 \\
\hline$C S-11 \sim 14$ & CS cv Liu Ben Cheng & Liu Ben Cheng & Huangyan, Zhejiang; 2012 & 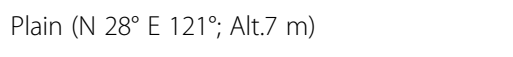 \\
\hline CS-15 & CS cv Hamlin & Hamlin & Huangyan, Zhejiang; 2012 & Plain (N 28 E $121^{\circ}$; Alt.7 m) \\
\hline CS-16 & CS cv Jin Cheng & Jin Cheng & Huangyan, Zhejiang; 2012 & 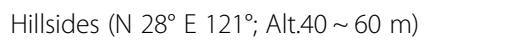 \\
\hline CS-17 & CS cv Navel orange & Navel orange & Huangyan, Zhejiang; 2012 & 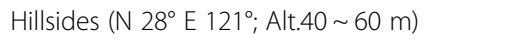 \\
\hline CS-18 21 & CS cv Delta Valencia & Delta Valencia & Huangyan, Zhejiang; 2012 & 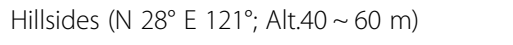 \\
\hline \multicolumn{5}{|c|}{ Poncirus trifoliate Raf. } \\
\hline PT-1 & PT & Trifoliate orange & Jiangjin, Sichuan; 2012 & 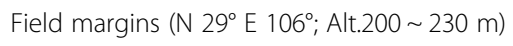 \\
\hline PT-2 & PT & Trifoliate orange & Huangyan, Zhejiang; 2012 & 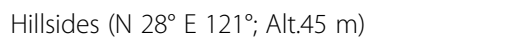 \\
\hline PT-3 $\sim 4$ & PT & Trifoliate orange & Huangyan, Zhejiang; 2012 & 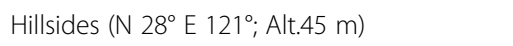 \\
\hline
\end{tabular}

temperature was varied from $50^{\circ} \mathrm{C}$ ( 1 min held) to $140^{\circ} \mathrm{C}$ ( $1 \mathrm{~min}$ held) at $5^{\circ} \mathrm{C} / \mathrm{min}$, and then from $140^{\circ} \mathrm{C}$ to $200^{\circ} \mathrm{C}$ (3 min held) at $10^{\circ} \mathrm{C} / \mathrm{min}$. The injector and interface temperatures were held at $250^{\circ} \mathrm{C}$. Mass spectra in the electron impact mode (EI-MS) were generated at $70 \mathrm{eV}$. The ion source temperature was held at $250^{\circ} \mathrm{C}$. The scanning time was $0.5 \mathrm{sec}$ over a range of $\mathrm{m} / z$ 45-450. An oil sample of $1 \mu \mathrm{L}$ was injected in the split mode injection (split ratio, 60:1).

\section{Identification and quantitative determination}

The volatile components were tentatively identified based on linear retention index (RI) and by the comparison of mass spectra with MS data of reference compounds. The linear retention indices were determined for all constituents by using a homologous series of $n$-alkanes $\left(\mathrm{C}_{8}-\mathrm{C}_{20}\right)$. The components were identified by comparison of their mass spectra with those of the NIST05 and NIST05S mass spectral library and further 
confirmed by comparison with the literature values $[19,25]$. The relative proportions of the essential oil constituents were expressed as percentages obtained by peak area normalization with all relative response factors being taken as one. The samples were run in triplicate.

\section{Statistical analysis}

The GC-MS data of different species of Zhishi samples were analyzed to identify potential discriminant variables. Multivariate statistical analyses, including unsupervised PCA and supervised partial least squares-discriminant analysis (PLS-DA), were performed using the SAS 9.1.3 statistical package (order no. 195557). PCA was used to observe the natural interrelationship among the chemical components for each of the four Citrus species. Analysis of variance was employed to identify those volatiles that would be most differentiating among the four species. Furthermore, the corresponding loading plot was used to interpret the variations among the samples. The critical $p$ value for all analyses in this study was set to 0.05 .

\section{Conclusion}

GC-MS coupled with multivariate statistical analysis of volatiles from different Citrus species, revealed a diverse volatile distribution among $C S, C J$ and $P T$. However, the volatiles contained in $C A$ exhibited less specificity; therefore, they could not be separated from the other three species. The PCA scores plot of $C S, C J$ and $P T$ appeared as widely distinct clustering. The chemical compounds accountable for the different volatile profiles of the three species are germacrene $D$, caryophyllen, $\beta$-myrcene, $\beta$ elemene, $\alpha$-farnesene, $\mathrm{p}$-mentha-1, 4(8)-diene, limonene, $(+)$-4-carene, $\beta$-pinene, $\gamma$-terpinene, $\alpha$-thujene, $p$-cymen2 -ol and $\alpha$-pinene, all of which are significantly up- or down- regulated in the different three species. In short, sesquiterpene compounds could serve as characteristic markers for $P T$. The content of some monoterpene compounds could be used as taxonomic markers between $C S$ and $C J$. This work demonstrates the variations of essential oils among different species of Citrus fruits and is of great significance in the pharmacological and clinical use of Zhishi.

\section{Additional file}

Additional file 1: Table S1. Identifications of Zhishi volatile in Citrus sinensis Osbeck and its cultivars. Table S2. Identifications of Zhishi volatile in Citrus junos Sieb. ex Tanaka. Table S3. Identifications of Zhishi volatile in Poncirus aurantium L. and its cultivars Table S4. Identifications of Zhishi volatile in Poncirus trifoliate Raf. Table S5. PCA loadings plot scores of all the GC-MS signals of the three species of Zhishi samples: 15 samples from CJ, 21 samples from $C J$ and 4 samples from $P T$.

\section{Abbreviations}

GC-MS: Gas chromatography-mass spectrometry; CS: Citrus sinensis; CJ: C. junos Sieb. ex Tanaka; CA: C. aurantium L; PT: Poncirus trifoliate Raf; PCA: Principal component analysis; Rl: Retention index; PLS-DA: Partial least squaresdiscriminant analysis; PC1: Principal component 1; PC2: Principal component 2; El-MS: Electron impact mode.

\section{Competing interests}

The authors declare that they have no competing interests.

\section{Authors' contributions}

$A L$ provided the concept and designed the study. $Y L$ and $Z L$ conducted the analyses and wrote the manuscript. CW, QZ, CL, ZS, ZN, SZ and XM participated in the research. All authors have read and approved the final manuscript.

\section{Acknowledgment}

This study was financially supported by the National Science Foundation of China (Project No. 30772726, No. 30825047 and No. 81001623).

\section{Author details}

${ }^{1}$ School of Chinese Materia Medica, Beijing University of Chinese Medicine, Beijing Municipal Key Laboratory for Basic Research of Chinese Medicine, Beijing 100102, China. ${ }^{2}$ Institution of Basic Theory, China Academy of Chinese Medical Sciences, Beijing 100700, China. ${ }^{3}$ Institute of Basic Research in Clinical Medicine, China Academy of Chinese Medical Sciences, Beijing 100700, China. ${ }^{4}$ School of Chinese Medicine, Hong Kong Baptist University, Hongkong, China.

Received: 15 December 2013 Accepted: 19 March 2014

Published: 3 April 2014

\section{References}

1. González ME, Domínguez PR, Moreno DA, García VC: Natural bioactive compounds of Citrus limon for food and health. J Pharm Biomed Anal 2010, 51:327-345.

2. Moufida S, Marzouk B: Biochemical characterization of blood orange, sweet orange, lemon, bergamot and bitter orange. Phytochemistry 2003, 62:1283-1289

3. Viuda MM, Ruiz NY, Fernandez LJ, Perez AJ: Antifungal activity of lemon (Citrus lemon L.), mandarin (Citrus reticulata L.), grapefruit (Citrus paradisi L.) and orange (Citrus sinensis L.) essential oils. Food Control 2008, 19:1130-1138

4. Burt S: Essential oils: their antibacterial properties and potential applications in foods-a review. Int J Food Microbiol 2004, 94:223-253.

5. Gaunt LF, Higgins SC, Hughes JF: Interaction of air ions and bactericidal vapours to control micro-organisms. J App/ Microbiol 2005, 99:1324-1329.

6. Sharma N, Tripathi A: Fungitoxicity of the essential oil of Citrus sinensis on post-harvest pathogens. World J Microbiol Biotechnol 2006, 22:587-593.

7. Casabianca H, Graff JB: Separation of linalyl acetate enantiomers: Application to the authentication of bergamot food products. J High Resolution Chromatogr 1994, 17:184-186.

8. Fernández L, Zhi N, Aleson CL, Pérez AJA, Kuri V: Antioxidant and antibacterial activities of natural extracts: application in beef meatballs. Meat Sci 2005, 69:371-380.

9. Depasquale F, Siragusa M, Abbate L, Tusa N, Depasquale C, Alonzo G: Characterization of five sour orange clones through molecular markers and leaf essential oils analysis. Sci Hortic 2006, 109:54-59.

10. Lan-Phi NT, Shimamura T, Ukeda H, Sawamura M: Chemical and aroma profiles of yuzu (Citrus junos) peel oils of different cultivars. Food Chem 2009, 115:1042-1047.

11. Jabalpurwala FA, Smoot JM, Rouseff RL: A comparison of citrus blossom volatiles. Phytochemistry 2009, 70:1428-1434.

12. Xie ZW: Research on duration and changes of Zhishi and Zhiqiao as ancient and present drugs. Res Tradit Chinese Med 1991, 1:19-22.

13. Cai YP, Chen YG, Fan CS: Studies on Chinese drugs Zhiqiao and Zhishi origin investigation and merchandise identification. China J Chinese Material Med 1999, 24:259-262.

14. The Pharmacopoeia Committee of China: Chinese Pharmacopoeia, 2010. Beijing: People's Medical Publishing House; 2010:172. 
15. Hao Q, Li Y: The effect and mechanism of different kinds of Bupleurum and Citrus on gastro-intestinal motility. Chinese J Nat Med 2002, 4:138-140.

16. Li Y, Chen S, Li Y, Xu B: Effect of each component of Shao-Yao-Gan-CaoTang and Si-Ni-San on gastricemptying and smali intestinal propulsive functions. Chinese J Digestion 1996, 16:18-21.

17. Liu ZL, Liu YY, Wang C, Song ZQ, Zha QL, Lu C, Wang C, Lu AP: Discrimination of Zhishi from different species using RRLC-DAD/UV coupled with multivariate statistical analysis. J Med Plants Res 2012. doi:10.5897/JMPR11.1504

18. Gao SY, Zheng HJ: GC Analysis of the Volatile Oil from the Fruit Peel and Immature Fruit of Citrus. Chinese J Pharmaceut Anal 1986, 6:83.

19. Liao FX, Sun GY, Yang ZB, Xia ZN: Chemical composition and antimicrobial activity of essential oil of Zhishi. Chinese Tradit Herbal Drugs 2004, 35:20-22.

20. Liao FX, Xin LT, Chen H, Xia ZN: Analysis of the constituents of volatile Oil from fructus aurantii immaturus and fructus aurantii as traditional chinese medicine. J Chongqing Univ 2004, 27:38-40.

21. Pan $X$, Liang M: Analysis of essential Oil from fructus ponciri trifoliatae immaturi by GC-MS. Tradit Chinese Drug Res Clin Pharmacol 2004, 15:415-416.

22. Bermejo A, Llosa MJ, Cano A: Analysis of bioactive compounds in seven citrus cultivars. Food Sci Techn Intern 2011, 17:55-62.

23. Menichini F, Tundis R, Bonesi M, de Cindio B, Loizzo MR, Conforti F, Statti GA, Menabeni R, Bettini R, Menichini F: Chemical composition and bioactivity of Citrus medica L. cv. Diamante essential oil obtained by hydrodistillation, cold-pressing and supercritical carbon dioxide extraction. Nat Prod Res 2011, 25:789-799.

24. Shaw PE: Review of quantitative analyses of citrus essential oils. J Agr Food Chem 1979, 27:246-257.

25. Zhao Y: Study on the Chromatography Fingerprint of Zhishi and Zhiqiao. In Research Center for Modernization of Traditional Chinese Medicines. Central South University: MHA Thesis; 2005.

doi:10.1186/1752-153X-8-22

Cite this article as: Liu et al: Study on essential oils from four species of Zhishi with gas chromatography-mass spectrometry. Chemistry Central Journal 2014 8:22.

\section{Publish with ChemistryCentral and every scientist can read your work free of charge \\ "Open access provides opportunities to our colleagues in other parts of the globe, by allowing anyone to view the content free of charge." \\ W. Jeffery Hurst, The Hershey Company. \\ - available free of charge to the entire scientific community \\ - peer reviewed and published immediately upon acceptance \\ - cited in PubMed and archived on PubMed Central \\ - yours - you keep the copyright \\ Submit your manuscript here: \\ http://www.chemistrycentral.com/manuscript/<smiles>c1ccccc1</smiles> \\ Chemistry Central}

